\title{
Multiple-Grid Convergence Acceleration of Viscous and Inviscid Flow Computations
}

Gary M. Johnson

Lewis Research Center

Cleveland, Ohio

\section{LIBRAEY GDPY \\ 16?: 105 \\ LANGLEY RESEARCH CENTER LIBRARY, NASA \\ HAMPTON, VIRGINIA}

Prepared for the

International Multigrid Conference

organized by the Institute for Computational Studies

at Colorado State University and the Gesellschaft

für Mathematik und Datenverarbeitung

cosponsored by AFOSR and NASA

Copper Mountain, Colorado, April 6-8, 1983 


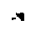


Multiple-Grid Convergence Acceleration of

Viscous and Inviscid Flow Computations

\author{
Gary M. Johnson \\ National Aeronautics and Space Administration \\ Lewis Research Center \\ Cleveland, Ohio
}

SUMMARY

A multiple-grid algorithm for use in efficiently obtaining steady solutions to the Euler and Navier-Stokes equations is presented. The convergence of a simple, explicit fine-grid solution procedure is accelerated on a sequence of successively coarser grids by a coarse-grid information propagation method which rapidly eliminates transients from the computational domain. This use of multiple-gridding to increase the convergence rate results in substantially reduced work requirements for the numerical solution of a wide range of flow problems. Computational results are presented for subsonic and transonic inviscid flows and for laminar and turbulent, attached and separated, subsonic viscous flows. Work reduction factors as large as eight, in comparison to the basic fine-grid algorithm, have been obtained. Possibilities for further performance improvement are discussed.

\title{
INTRODUCTION
}

When steady solutions to the Euler or Navier-Stokes equations are sought, it is common practice to compute them as temporal asymptotes to the unsteady equations of motion, subject to steady boundary conditions and the hypothesis that such solutions exist. This is done because the unsteady equations are either purely hyperbolic, in the case of the Euler equations, or hyperbolic - parabolic, for the Navier-Stokes equations, and are thus amenable to numerical solution by robust time-marching procedures. Such procedures are relatively easy to implement, readily capture weak solutions and, to the extent that the computation is time-accurate, allow a physical interpretation of the transient behavior, which may prove valuable in analyzing the performance of the algorithm.

On the other hand, relaxation procedures for solving the steady versions of the Euler or Navier-Stokes equations are a topic of current research and have not as yet resulted in production algorithms. Attention has focused primarily on the steady Euler equations, where the research in progress includes the work of Bruneau, Chattot, Laminie and Guiu-Roux (1982) and Johnson (1982b).

Both explicit and implicit time-marching procedures are presently in widespread use. Explicit methods, such as the MacCormack (1969) scheme, are simple, easily vectorizable and allow a good deal of flexibility in the treatment 
of boundary conditions. Their largest shortcoming lies in their conditional stability, which may place rather severe limitations on the time step size permissible on a given grid. When only a steady solution is sought and the accurate resolution of transients is of no consequence, explicit methods may consequently exhibit poor convergence rates.

One remedy to the slow convergence of explicit schemes which has been investigated in recent years by Beam and Warming (1976), Steger (1977), MacCormack (1981), Lerat, Sides and Daru (1982) and others, is the construction of implicit methods. These methods, at the expense of a higher operations count, are generally unconditionally linearly stable and hopefully permit time steps to be taken as large as is consistent with accuracy requirements. In pnactice, large time steps may excite nonlinear instabilities and the choice of boundary condition implementation may introduce a stability limit.

Another approach is to maintain the explicit nature of the integration scheme but to aband on the temporal accuracy requirement in favor of the introduction of convergence acceleration techniques, such as numerical methods with stability bounds in excess of the CFL limit or enhanced temporal damping procedures. Such techniques are exhibited in the work of Essers (1978), Viviand (1981), Jameson, Schmidt and Turkel (1981) and Rizzi and Eriksson (1981).

Recently, efforts have been made at adapting multigrid techniques, which have shown substantial promise for the fast numerical solution of elliptic partial differential equations, to the Euler equations. Here, the nonlinear, nonelliptic nature of the equations, the existence of weak solutions and the fact that a coupled system of equations, rather than a single scalar equation, must be solved have been major obstacles. Jespersen (1983) has made notable progress in this direction.

The present work, which is based on a concept introduced by $\mathrm{Ni}$ (1981), uses a sequence of successively coarser grids to accelerate the convergence of an explicit fine-grid solution procedure. In this general sense, it is a multiple-grid method. However, the details of its implementation are a good deal simpler than is the case with the conventional multigrid approach. The coarse-grid acceleration scheme presented here is quite modular. It may be used without modification in conjunction with a number of different fine-grid solution procedures and with any set of flow equations in the hierarchy ranging from the Euler equations to the full Navier-Stokes equations.

\section{EQUATIONS OF MOTION}

The nondimensional equations of motion may be written in conservation-law form as

$$
q_{t}=-\left(F_{x}+G_{y}\right)
$$

where, for the full Navier-Stokes equations,

$$
\mathrm{F} \equiv \mathrm{f}-\mathrm{Re}^{-1} \mathrm{r} \quad \mathrm{G} \equiv \mathrm{g}-\mathrm{Re}^{-1} \mathrm{~s}
$$


while, for their thin-layer version,
$\mathrm{F} \equiv \mathrm{f}$
$G \equiv g-\operatorname{Re}^{-1 \tilde{s}}$

and, for the Euler equations,
$\mathrm{F} \equiv \mathrm{f}$
$G \equiv g$

where:

$$
\begin{aligned}
& q=\left[\begin{array}{c}
\rho \\
\rho u \\
\rho v \\
E
\end{array}\right] \quad f=\left[\begin{array}{l}
\rho u \\
\rho u^{2}+p \\
\rho u v \\
(E+p) u
\end{array}\right] \quad g=\left[\begin{array}{l}
\rho v \\
\rho u v \\
\rho v^{2}+p \\
(E+p) v
\end{array}\right] \\
& r=\left[\begin{array}{l}
0 \\
\tau_{x x} \\
\tau_{x y}
\end{array}\right] s=\left[\begin{array}{l}
0 \\
\tau_{x y} \\
\tau_{y y}
\end{array}\right. \\
& \left.\kappa \operatorname{Pr}^{-1}(\gamma-1)^{-1}\left(a^{2}\right)_{x}+u \tau_{x x}+v \tau_{x y}\right] \quad\left[\operatorname{Pr}^{-1}(\gamma-1)^{-1}\left(a^{2}\right)_{y}+u \tau_{x y}+v \tau_{y y}\right. \\
& \tilde{\mathbf{s}}=\left[\begin{array}{l}
0 \\
\mu u_{y} \\
(\lambda+2 \mu) v_{y} \\
\kappa \operatorname{Pr}^{-1}(\gamma-1)^{-1}\left(a^{2}\right)_{y}+\mu\left(u u_{y}+2 v v_{y}\right)+\lambda v v_{y}
\end{array}\right] \\
& \tau_{\mathrm{xx}}=(\lambda+2 \mu) \mathrm{u}_{\mathrm{x}}+\lambda \mathrm{v}_{\mathrm{y}} \\
& \tau_{x y}=\mu\left(u_{y}+v_{x}\right) \\
& \tau_{y y}=(\lambda+2 \mu) v_{y}+\lambda u_{x}
\end{aligned}
$$

Here $\rho, u, v, p, a$ and $E$ are respectively density, velocity components in the $x-$ and $y$-directions, pressure, sound speed and total energy per unit volume. The total energy per unit volume may be expressed as

$$
E=\rho\left(e+\frac{1}{2}\left(u^{2}+v^{2}\right)\right)
$$

where the specific internal energy, $e$, is related to the pressure and density by the simple law of a calorically perfect gas

$$
p=(\gamma-1) \rho e
$$

with $\gamma$ denoting the ratio of specific heats. The coefficient of thermal conductivity, $\kappa$, and the viscosity coefficients, $\lambda$ and $\mu$, are assumed to be 
functions only of temperature. Furthermore, by invoking Stokes' assumption of zero bulk viscosity, $\lambda$ may be expressed in terms of the dynamic viscosity $\mu$ as

$$
\lambda=-\frac{2}{3} \mu
$$

Re and $\operatorname{Pr}$ denote the Reynolds and Prandt numbers, respectively.

Although, for simplicity, the equations of motion are presented here written in Cartesian coordinates, Viviand (1974) has shown that their strong conservation law form may be maintained under an arbitrary time-dependent transformation of coordinates. Explicit detail concerning the generalized coordinate version of these equations which is employed in the computations to be discussed subsequently has been provided by Steger (1977) and need not be repeated here.

We note that the thin-layer approximation, in the words of Baldwin and Lomax (1978), "... evolves directly from a realistic assessment of what is really being computed in a typical high Reynolds number Navier-Stokes simulation." A highly stretched mesh is used to resolve the large flow gradients normal to the vorticity-generating surface. Consequently, because of limitations on computer capacity, the diffusion terms involving derivatives parallel to the surface are not resolved well enough to merit their computation.

Similar viscous terms are also neglected in the classical boundary layer approximation. However, while the boundary layer approximation replaces the normal momentum equation with the assumption that the normal pressure gradient is zero across the viscous layer, all momentum equations are retained in the thin-layer approximation and no assumptions are made concerning the pressure. Consequent1y, the separation point is not a singularity of the thin-1ayer model equations nor do the problems associated with matching a boundary layer solution to an inviscid outer flow occur when they are used.

In practice, the thin-layer assumption is implemented by using a body-fitted coordinate system and neglecting the viscous terms in the coordinate direction along the body. For Cartesian coordinates, with $x$ representing the body-conforming coordinate, the thin-layer version of the Navier-Stokes equations is as given above.

The effects of turbulence are simulated by means of a two-layer algebraic eddy viscosity model. In the stress terms of the Navier-Stokes equations, the coefficient of dynamic viscosity, $\mu$, is replaced by $\mu+\mu_{t}$, where $\mu_{t}$ is the coefficient of eddy viscosity. Similarly, in the heat flux terms, the coefficient of thermal conductivity, $k$, is replaced by $k+c_{p} \mu_{t} / \operatorname{Pr}_{t}$, where $\operatorname{Pr}_{t}$ is the turbulent Prandt1 number. The eddy viscosity is determined by the method of Baldwin and Lomax (1978) which is patterned after that of Cebeci (1970) with modifications to avoid the necessity of finding the boundary layer edge. Details may be found in the work of Baldwin and Lomax.

\section{FINE-GRID SOLUTION PROCEDURE}

The fine-grid integration scheme employed in this work is the two-step Lax-Wendroff method known as the MacCormack (1969) scheme. We observe that 
schemes of Lax-Wendroff type may be arrived at intuitively by using Taylor's theorem to write the approximation:

$$
\delta q=\Delta t q_{t}+\frac{\Delta t^{2}}{2} q_{t t}
$$

where we define the "correction" to q such that

$$
\delta q \equiv q(t+\Delta t)-q(t)
$$

Since we seek solutions to Eqn. (1), time derivatives may be expressed as space derivatives:

$$
\begin{aligned}
& q_{t}=-\left(F_{x}+G_{y}\right) \\
& q_{t t}=\left[A\left(F_{x}+G_{y}\right)\right] x+\left[B\left(F_{x}+G_{y}\right)\right] y
\end{aligned}
$$

where $A$ and $B$ are the Jacobian matrices:
$A \equiv \partial F / \partial q$
$B \equiv \partial G / \partial q$

Substitution into Eqn.(2) results in:

$$
\begin{aligned}
& \delta q=-\Delta t\left(F_{x}+G_{y}\right) \\
& +\frac{\Delta t^{2}}{2}\left\{\left[A\left(F_{x}+G_{y}\right)\right] x+\left[B\left(F_{x}+G_{y}\right)\right] y\right.
\end{aligned}
$$

\section{One-Step Methods}

Second-order accurate spatial discretization of Eqn. (3) yields a one-step Lax-Wendroff method. Prior to discussing two-step schemes, we derive the one-step Lax-Wendroff fine-grid solution procedure used by $\mathrm{Ni}$ (1981). This is a necessary prerequisite to the development of the coarse-grid acceleration scheme.

If we make the following finite-volume type approximations:

$$
\begin{aligned}
\left(F_{x}+G_{y}\right)_{i, j} & =\frac{1}{8 \Delta x}\left[\left(F_{i+1, j+1}+2 F_{i+1, j}+F_{i+1, j-1}\right)\right. \\
& \left.-\left(F_{i-1, j+1}+2 F_{i-1, j}+F_{i-1, j-1}\right)\right] \\
& +\frac{1}{8 \Delta y}\left[\left(G_{i-1, j+1}+2 G_{i, j+1}+G_{i+1, j+1}\right)\right. \\
& \left.-\left(G_{i-1, j-1}+2 G_{i, j-1}+G_{i+1, j-1}\right)\right]
\end{aligned}
$$


and define the "change" in q at cell centers such that:

$$
\begin{aligned}
\dot{q}_{i+\frac{1}{2}, j+\frac{1}{2} \equiv} & -\frac{\Delta t}{2 \Delta x}\left[\left(F_{i+1, j}+F_{i+1, j+1}\right)-\left(F_{i, j}+F_{i, j+1}\right)\right] \\
& -\frac{\Delta t}{2 \Delta y}\left[\left(G_{i, j+1}+G_{i+1, j+1}\right)-\left(G_{i, j}+G_{i+1, j}\right)\right]
\end{aligned}
$$

it then follows that a discrete approximation to the first-order term in Eqn. (3) may be written as

$$
\begin{aligned}
-\Delta t\left(F_{x}+G_{y}\right)_{i, j}=\frac{1}{4} & {\left[\begin{array}{c}
\Delta q_{i-\frac{1}{2}, j-\frac{1}{2}}+\Delta q \\
i-\frac{1}{2}, j+\frac{1}{2}
\end{array}\right.} \\
& \left.+\Delta q_{i+\frac{1}{2}, j+\frac{1}{2}}+\Delta q_{i+\frac{1}{2}, j-\frac{1}{2}}\right]
\end{aligned}
$$

Consistent with the above approximations and definition, we may write the approximation:

$$
-\Delta t\left(F_{x}+G_{y}\right)_{i+\frac{1}{2}, j+\frac{1}{2}}=\Delta q_{i+\frac{1}{2}, j+\frac{1}{2}}
$$

This motivates the definitions:

$$
\begin{aligned}
& \Delta F_{i+\frac{1}{2}, j+\frac{1}{2}} \equiv A_{i+\frac{1}{2}, j+\frac{1}{2}}^{\Delta q_{i+\frac{1}{2}}, j+\frac{1}{2}} \\
& G_{i+\frac{1}{2}, j+\frac{1}{2}} \equiv B_{i+\frac{1}{2}, j+\frac{1}{2}}^{\Delta q_{i+\frac{1}{2}}, j+\frac{1}{2}}
\end{aligned}
$$

If we then approximate the second-order terms in Eqn. (3) such that:

$$
\begin{aligned}
-\Delta t & \left\{\left[A\left(F_{x}+G_{y}\right)\right]_{x}\right\}_{i, j}= \\
& \frac{1}{2 \Delta x}\left[F_{i+\frac{1}{2}, j+\frac{1}{2}}+\Delta F_{i+\frac{1}{2}, j-\frac{1}{2}-\Delta F_{i-\frac{1}{2}, j-\frac{1}{2}}-\Delta F} i-\frac{1}{2}, j+\frac{1}{2}\right]
\end{aligned}
$$




$$
\begin{aligned}
& -\Delta t\left\{\left[B\left(F_{x}+G_{y}\right)\right]_{x}\right\}_{i, j}= \\
& \frac{1}{2 \Delta y}\left[G_{i+\frac{1}{2}, j+\frac{1}{2}}+\Delta G_{i-\frac{1}{2}, j+\frac{1}{2}}-\Delta G_{i-\frac{1}{2}, j-\frac{1}{2}}-\Delta G i+\frac{1}{2}, j-\frac{1}{2}\right]
\end{aligned}
$$

we may combine Eqns. (5) and (6) to yield:

$$
\begin{aligned}
\delta q_{i, j} & =\frac{1}{4}\left[\Delta q+\frac{\Delta t}{\Delta x} \Delta F+\frac{\Delta t}{\Delta y} \Delta G\right]_{i-\frac{1}{2}, j-\frac{1}{2}} \\
& +\frac{1}{4}\left[\Delta q+\frac{\Delta t}{\Delta x} \Delta F-\frac{\Delta t}{\Delta y} \Delta G\right]_{i-\frac{1}{2}, j+\frac{1}{2}} \\
& +\frac{1}{4}\left[\Delta q-\frac{\Delta t}{\Delta x} \Delta F-\frac{\Delta t}{\Delta y} \Delta G\right]_{i+\frac{1}{2}, j+\frac{1}{2}} \\
& +\frac{1}{4}\left[\Delta q-\frac{\Delta t}{\Delta x} \Delta F+\frac{\Delta t}{\Delta y} \Delta G\right]_{i+\frac{1}{2}, j-\frac{1}{2}}
\end{aligned}
$$

Eqns. (4) and (7) constitute the one-step Lax-Wendroff method used as a basic integration scheme by $\mathrm{Ni}$. He gives the following heuristic interpretation to these equations: the first calculates the change in $q$ occurring in a control volume during the increment $\Delta t$, while the second distributes the effects of the changes occurring in four nearest-neighbor control volumes to their common central nodal point. There they are combined to form the correction to the vector of conservation variables, as illustrated in Fig. 1. This interpretation motivates the construction of the coarse-grid acceleration scheme to be discussed subsequently.

Notice that Ni's scheme may also be considered to be a two-step scheme with a full time increment predictor defined by Eqn. (4) and a corrector defined by Eqn. (7). However, such an interpretation would not be consistent with the general practice of avoiding the computation of Jacobian matrices in two-step schemes.

Two-Step Methods

Following Richtmyer (1962) many two-step Lax-Wendroff schemes have been developed. They have superseded the one-step schemes by virtue of their lower operations counts. MacCormack's method is a particularly popular and efficient member of this class. The forward predictor - backward corrector version of this 
method may be written as

$$
\begin{aligned}
\Delta q_{i, j}= & -\frac{\Delta t}{\Delta x}\left(F_{i+1, j}^{n}-F_{i, j}^{n}\right)-\frac{\Delta t}{\Delta y}\left(G_{i, j+1}^{n}-G_{i, j}^{n}\right) \\
\delta q_{i, j}= & -\frac{\Delta t}{2 \Delta x}\left[\begin{array}{c}
\left(F_{i+1, j}^{n}-F_{i, j}^{n}\right)+\left(\tilde{F}_{i, j}-\tilde{F}_{i-1, j}\right)
\end{array}\right] \\
& -\frac{\Delta t}{2 \Delta y}\left[\left(G_{i, j+1}^{n}-G_{i, j}^{n}\right)+\left(\tilde{G}_{i, j}-\tilde{G}_{i, j-1}\right)\right]
\end{aligned}
$$

where:

$$
\begin{aligned}
& \Delta q_{i, j}=\tilde{q}_{i, j}-q_{i, j}^{n} \\
& \delta q_{i, j}=[q(t+\Delta t)-q(t)]_{i, j} \\
& \tilde{F}_{i, j}=F\left(\tilde{q}_{i, j}\right) \\
& \tilde{G}_{i, j}=G\left(\tilde{q}_{i, j}\right)
\end{aligned}
$$

First derivatives in the viscous terms are backward differenced in the predictor and forward differenced in the corrector.

The two-step methods of Burstein (1966) and Lapidus (1967) were also examined during the course of this work. Although they are spatially symmetric, their use resulted in no observable accuracy advantage over MacCormack's method, for the test cases considered. Furthermore, they are computationally less efficient than MacCormack's method. Details are contained in Johnson (1982a).

This approach to solving fluid flow problems is quite robust and has been in widespread and successful use for some time, both for the time-accurate computation of unsteady flow and for the time-asymptotic solution of steady flow problems. In the latter case, where accurate resolution of physical transients is not required, the numerical stability limitation inherent in these explicit methods may severely restrict the speed of their convergence to the steady state. Providing a method to accelerate convergence in this case is the objective of this work.

\section{COARSE-GRID ACCELERATION SCHEME}

Given the fine-grid corrections, which may be computed by any one- or two-step Lax-Wendroff scheme, we wish to use successively coarser grids to propagate these corrections throughout the computational domain, thus accelerating convergence to the steady state while maintaining the accuracy determined by the fine-grid discretization. Given a basic fine grid with the number of points in each direction expressible as $n\left(2^{p}\right)+1$ for $p$ and $n$ integers such that $p \geq 0$ and $\mathrm{n} \geq 2$, where $\mathrm{p}$ is the number of grid coarsenings and $\mathrm{n}$ is the number of 
coarsest-grid intervals, let successively coarser grids be defined by successive deletion of every other point in each coordinate direction.

\section{Ful1 Coarse-Grid Scheme}

The full coarse-grid acceleration scheme, as illustrated in Fig. 2, replaces the computation of coarse grid changes by Eqn. (4) with a restriction of the latest fine-grid correction. This restricted fine-grid correction is then distributed according to a coarse-grid version of Eqn. (7) to obtain a coarse-grid correction. This coarse-grid correction is, in turn, prolonged to the fine grid to become the new fine-grid correction. One time-cycle of the multiple-grid scheme is composed of an application of some Lax-Wendroff method on the fine grid followed by an application of the coarse-grid propagation scheme to each successively coarser grid. The flow of information in this process is depicted in Fig. 3.

In the basic integration scheme, a change at one grid point affects only its nearest neighbors while, in a k-level multiple-grid scheme, the same change affects all points up to $2^{\mathrm{k}-1}$ mesh spacings distant. Furthermore, since the change is always determined by information from the fine grid and simply propagated by the distribution formulae for coarser grids, fine grid accuracy is maintained.

This concept for convergence acceleration was introduced by $\mathrm{Ni}$ for use in conjunction with his one-step Lax-Wendroff scheme, as described above. He illustrated its utility by solving the homoenthalpic two-dimensional Euler equations. Johnson (1982a) extended its applicability to the class of two-step Lax-Wendroff schemes and solved the full two-dimensional Euler equations.

\section{Convective Coarse-Grid Scheme}

In Johnson (1982c), consideration of the physical processes being modelled in a viscous flow computation led to the formulation of an alternative coarse-grid scheme. Dissipative effects have a local character and their influence need not be taken into account in the construction of coarse-grid distribution formulae. Rather, it is the convective terms, with their global character, which are the key element in coarse-grid propagation. Hence, a coarse-grid scheme for viscous flow computations may be formulated on the basis of the inviscid equations of motion. Such a convective coarse-grid scheme is inherently more efficient than the full coarse-grid scheme because of the diminished computational effort associated with forming the Jacobian matrices of the Euler flux vectors rather than those of the viscous flux vectors. An additional benefit is that the convective coarse-grid scheme leads to a multiple-grid convergence acceleration procedure which is independent of the nature of the dissipative terms retained in the viscous model equations. That is to say: the coarse-grid scheme based on the Euler equations may be employed, without modification, to accelerate the convergence of viscous flow computations based on the Navier-Stokes equations, the thin-layer equations, or any other viscous model equations which contain the full inviscid Euler equations.

The correctness of the heuristic physical reasoning used in the formulation of the convective coarse-grid scheme is supported by the computational results for viscous flow to be presented subsequently. 
COMPUTATIONAL RESULTS

To investigate the robustness of the coarse-grid acceleration scheme and to explore the generality of its applicability, a large number of computational experiments have been performed. A representative sampling of those computations is reported here.

The full Euler equations have been solved for both subsonic and transonic flow. The thin-layer version of the Navier-Stokes equations has been solved for attached and separated, laminar and turbulent, subsonic flows. A11 computations have been performed in two dimensions. Extension of the technique to the full Navier-Stokes equations or to three dimensions presents no essential difficulties and should, in fact, yield greater advantage than is illustrated by the present results.

\section{Problem Specification}

We consider the inviscid flow through an unstaggered cascade of bicircular arc airfoils at zero angle of attack, as illustrated in Fig. 4, and the viscous flow through a similar cascade of sting-mounted airfoils, as shown in Fig. 5. The boundary conditions used are also indicated in these Figures. At the upstream domain boundary, total pressure, total temperature and flow angle are specified. At the downstream boundary, the static pressure is fixed. Along inviscid latera1 boundaries, the tangency condition is applied, while, along solid walls, the no-slip condition is applied and the temperature specified. The values of the dependent variables on the domain boundaries are updated only during the fine-grid computations. This decouples the coarse-grid acceleration scheme from the details of boundary condition implementation. Uniform flow at the isentropic Mach number implied by the ratio of exit static pressure to upstream total pressure is used as an initial: state.

For the subcritical cases, the ratio of exit static pressure to upstream total pressure is 0.8430191 , yielding an isentropic upstream Mach number of 0.500 , while, for the supercritical cases a ratio of 0.7369520 is employed, resulting in an upstream Mach number of 0.675. In the viscous cases, the Reynolds numbers, based on cascade gap and critical speed, span the approximate range from $8.4 \times 10^{3}$ to $2.0 \times 10^{5}$.

Symmetry and periodicity are invoked to limit the size of the computational domain. All inviscid computations were carried out using the $65 \times 17$ node fine grid as shown in Fig. 6 and the coarser grids as indicated in Table I. As is also illustrated in Fig. 6, three different fine grids were employed in the viscous flow computations. All have $65 \times 33$ nodal points and have their transverse grid lines located at the same positions. They differ in the positioning of their lateral grid 1ines. These are smoothly stretched away from the solid boundary in a geometric progression, starting from the initial spacings indicated in Fig. 6 . These fine grids each allow the construction of four successively coarser grids, as shown in Table II. 
Inviscid Flow

The results of typical subcritical inviscid flow computations are illustrated in Fig. 7. The airfoils have a thickness-to-chord ratio of 0.200 . Isomach contours and the surface Mach number distributions along the upper and lower domain boundaries are shown. We observe that the fore-and-aft geometric symmetry is well preserved in the computed flow field.

All computations reported here are considered to be converged when the average absolute value of the unscaled fine-grid residual in $\rho$ u drops below $10^{-6}$, representing a decrease of approximately five decades from its original value. This convergence criterion is more than sufficient for engineering purposes and could be relaxed by at least two decades without any significant accuracy deterioration in the solution. The converged single- and multiple-grid results were, as should be expected, identical, confirming that the solution obtained is not a function of grid sequence length.

Based on the computational work required to produce a converged solution, we may estimate a multiple-grid work reduction factor and a corresponding optimal grid sequence length. We define the work reduction factor to be the ratio of the work required to produce a converged solution using a single fine grid, to the work required to produce the same result using that multiple-grid sequence length which minimizes the computational work. The results obtained are recorded in Table III. For the case at hand, a sequence of three grids produced a work reduction factor of 5.1 . The single- and optimal multiple-grid convergence histories are also shown in Fig. 7.

Supercritical results for the same thickness-to-chord ratio are shown in Fig. 8. Here, we observe that the shock is crisply resolved by the multiple-grid scheme although the explicit artificial viscosity term added to the algorithm for this purpose is only applied on the fine grid. We hasten to add that no artificial viscosity beyond that inherent in the basic multiple-grid algorithm was employed in either the inviscid or viscous subcritical computations reported here. A sequence of four grids produced a work reduction factor of 2.1 for this inviscid supercritical flow case.

Viscous Flow

Viscous flow computations have been performed for the combinations of Reynolds number, thickness-to-chord ratio and fine grid configuration indicated in Table III. A sampling of the results is illustrated in Figs. 9 through 12 . In each case, isomach contours, u-velocity profiles and the single- and optimal multiple-grid convergence histories are shown. Note that the contour levels displayed are not equally spaced and that the same levels were used in all illustrations. Nevertheless, they provide a good qualitative indication of the nature of the computed flowfields. More quantitative information is provided by the normalized u-velocity profiles. The u-velocity, normalized with its value at the domain top boundary and same streamwise station, is plotted as a function of relative distance from the airfoil chord line. Curves for every second streamwise station, starting with the airfoil leading edge and ending at the outflow boundary, are displayed. They are staggered in proportion to the spacing of their respective streamwise stations. 
The thickness-to-chord ratios employed ranged from 0.100 to 0.000 . Both laminar and turbulent flows have been computed. In the turbulent cases, the flow was tripped at the airfoil leading edge. Attached and separated cases were computed for both laminar and turbulent flow.

The work reduction factors obtained ranged from 1.7 to 8.2 and are recorded in Table III, together with the optimal grid sequence lengths. We note that Johnson (1982c) observed that although the work reduction factor and, possibly, the optimal grid sequence length decrease with increasing grid stretching, they do not appear to decrease with increasing Reynolds number.

\section{ALGORITHMIC CONSIDERATIONS}

During the course of this work, several issues bearing on the speed, computational efficiency, storage requirements and general applicability of this multiple-grid convergence acceleration scheme have come to mind. As most of these issues deserve a good deal more study, we let the following brief remarks suffice for the present.

\section{Boundary Condition Implementation}

As mentioned previously, boundary conditions are only enforced on the fine grid. This has the advantage of decoupling the coarse-grid acceleration scheme from both the physical and numerical nature of these boundary conditions. That is to say: the coarse-grid scheme always sees a Dirichlet problem. While this enhances the modularity of the coarse-grid scheme, it is not clear that such a boundary condition treatment serves to maximize the convergence acceleration of the resultant algorithm.

\section{Accelerator Linearization}

The computation of the Jacobian matrices used in the coarse-grid acceleration scheme has a non-trivial influence on the coarse-grid operations count and, hence, on the work reduction factor. We have found that the Jacobians need not be updated at each time cycle, but may be lagged by a substantial margin without any adverse effect on the resultant convergence history. In fact, in some cases this improves the convergence rate and, in so doing, raises the possibility of tailoring the Jacobian matrices to accelerate convergence rather than calculating them based on the actual state of the flow being computed.

\section{Storage Minimization}

Linearizing the coarse-grid acceleration scheme in the sense described above implies storing the Jacobian matrices rather than recomputing them based on the latest available flow state. Should this storage requirement constitute an unacceptable burden, it may be essentially eliminated by periodically storing information on the flow state at a small number of representative points and reconstructing a set of Jacobians from this information at each time cycle. Such a strategy would be in complete accord with our linearization ideas. 
Restriction and Prolongation Operators

In the present computations, injection is used as the restriction operator and linear interpolation is chosen as the prolongation operator. These choices may not be optimal for use on highly stretched grids. Better choices could increase both the optimal grid sequence length and the work reduction factor.

Coarse-Grid Formation Strategy

Similar consequences might result from an alternative coarse-grid formation strategy. Given the convective nature of the coarse-grid scheme used here, it is entirely plausible that, particularly in viscous flow computations, the coarser grids should have a more uniform structure than is obtained by successive deletion of every other grid line from a highly stretched fine grid.

\section{Coarse-Grid Propagation Strategy}

In distinction to conventional multi-gridding, the current algorithm cycles from the finest grid to the coarsest at each step to the solution procedure. Consequently, relatively many computations are performed on the finest grid. Computational expense could be reduced by developing an alternative coarse-grid propagation strategy which would spend more of its time at the coarser grid levels.

\section{Vectorization}

As both the fine-grid solution procedure and the coarse-grid acceleration scheme used here are explicit, the resultant multiple-grid algorithm may be readily vectorized. Suchvectorization is presently being performed and will be reported at a later date by Chima and Johnson (1983).

\section{Applicability of the Accelerator}

As the coarse-grid scheme uses a one-step Lax-Wendroff framework and as this concept was first employed by $\mathrm{Ni}$ to accelerate his one-step Lax-Wendroff fine-grid solution procedure, it is natural that the first generalization should be to the class of two-step Lax-Wendroff schemes. However, there is no compelling reason to expect that its applicability is limited to this class of methods. We expect that it may be used with a variety of explicit fine-grid solution procedures and, in fact, Stubbs (1983) has demonstrated its use with the implicit MacCormack (1981) scheme.

\section{CONCLUSIONS}

An explicit multiple-grid algorithm for the efficient solution of the steady Euler and Navier-Stokes equations has been presented.

The coarse-grid scheme used to accelerate convergence is compatible with all one- and two-step Lax-Wendroff algorithms. Here, it has been used in conjunction with MacCormack's method. 
The convective version of the coarse-grid scheme may be used with any set of flow equations in the hierarchy ranging from the Euler equations to the full Navier-Stokes equations. Here, we have used it with the thin-layer version of the Navier-Stokes equations.

Computational results have been presented for subsonic and transonic inviscid flows and for separated and attached, laminar and turbulent, subsonic viscous flows.

Multiple-gridding yielded work reduction factors ranging approximately from two to eight, over a fairly broad range of flow conditions. Ample margin remains for further performance improvements.

Given the encouraging results obtained to date, more comprehensive testing and more sophisticated applications of the inviscid and viscous flow convergence acceleration ideas presented here are planned.

\section{ACKNOWLEDGEMENTS}

The insightful suggestions and constructive criticism of my colleagues, Dr. Rodrick V. Chima and Dr. Robert M. Stubbs, are most gratefully acknowledged.

\section{REFERENCES}

Baldwin, B.S. and Lomax, H.: Thin-Layer Approximation and Algebraic Model for Separated Turbulent Flows. AIAA Paper 78-257, Jan. 1978.

Beam, R.M. and Warming, R.F.: An Implicit Finite-Difference Algorithm for Hyperbolic Systems in Conservation-Law Form. J. Comput. Phys., Vo1. 22, No. 1, Sep. 1976, pp. 87-110.

Bruneau, C.H., Chattot, J.J., Laminie, J. and Guiu-Roux, J.: Finite Element Least Square Method for Solving Full Steady Euler Equations in a Plane Nozzle. Proceedings of the Eighth International Conference on Numerical Methods in F1uid Dynamics, Lecture Notes in Physics, Vol. 170, Springer-Verlag, 1982, pp. 161-166.

Burstein, S.z.: Finite-Difference Calculations for Hydrodynamic Flows Containing Discontinuities. J. Comput. Phys., Vol. 1, No. 2, Nov. 1966, pp. 198-222.

Cebeci, T.: Calculation of Compressible Turbulent Boundary Layers with Heat and Mass Transfer. AIAA Paper 70-741, June 1970.

Chima, R.V. and Johnson, G.M.: Efficient Solution of the Euler and Navier-Stokes Equations with a Vectorized Multiple-Grid Algorithm.

AIAA Paper to appear, July 1983.

Essers, J.A.: Time-Dependent Methods for Mixed and Hybrid Steady Flows. von Karman Institute for Fluid Dynamics, Lecture Series 1978-4, Mar. 1978. 
Jameson, A., Schmidt, W. and Turkel, E.: Numerical Solutions of the Euler Equations by Finite Volume Methods Using Runge-Kutta Time-Stepping Schemes. AIAA Paper 81-1259, June 1981.

Jespersen, D.C.: A Multigrid Method for the Euler Equations. AIAA Paper 83-0124, Jan. 1983.

Johnson, G.M.: Multiple-Grid Acceleration of Lax-Wendroff Algorithms. NASA TM-82843, Mar. 1982a.

Johnson, G.M.: Relaxation Solution of the Full Euler Equations. Proceedings of the Eighth International Conference on Numerical Methods in Fluid Dynamics, Lecture Notes in Physics, Vo1. 170, Springer-Verlag, 1982b, pp. 273-279.

Johnson, G.M.: Convergence Acceleration of Viscous Flow Computations. NASA TM-83039, Oct. 1982c.

Lapidus, A.: A Detached Shock Calculation by Second-Order Finite Differences. J. Comput. Phys., Vo1. 2, No. 2, Nov. 1967, pp. 154-177.

Lerat, A., Sides, J. and Daru, V.: An Implicit Finite-Volume Method for Solving the Euler Equations. Proceedings of the Eighth International Conference on Numerical Methods in Fluid Dynamics, Lecture Notes in Physics, Vol. 170, Springer-Verlag, 1982, pp. 343-349.

MacCormack, R.W.: The Effect of Viscosity in Hypervelocity Impact Cratering. AIAA Paper 69-354, Apr. 1969.

MacCormack, R.W.: A Numerical Method for Solving the Equations of Compressible Viscous Flow. AIAA Paper 81-0110, Jan. 1981.

Ni, R.H.: A Multiple Grid Scheme for Solving the Euler Equations. AIAA Paper 81-1025, June 1981.

Richtmyer, R.D.: A Survey of Difference Methods for Non-Steady Fluid Dynamics. NCAR-TN-63-2, National Center for Atmospheric Research, Aug. 1982.

Rizzi, A. and Eriksson, L.E.: Transfinite Mesh Generation and Damped Euler Equation Algorithm for Transonic Flow Around Wing-Body Configurations. AIAA Paper 81-0999, June 1981.

Steger, J.L.: Implicit Finite Difference Simulation of Flow About Arbitrary Geometries with Application to Airfoils. AIAA Paper 77-665, June 1977.

Stubbs, R.M.: Multiple-Gridding of the Euler Equations with an Implicit Scheme. AIAA Paper to appear, July 1983.

Viviand, H.: Formes Conservatives des Equations de la Dynamique des Gaz. La Recherche Aérospatiale, No. 1, Jan.-Feb. 1974, pp. 65-66.

Viviand, H.: Pseudo-Unsteady Methods for Transonic Flow Computations. Proceedings of the Seventh International Conference on Numerical Methods in Fluid Dynamics, Lecture Notes in Physics, Vol. 141, Springer-Verlag, 1981, pp. 44-54. 
TABLE I. - INVISCID FLOW GRID DESCRIPTIONS

\begin{tabular}{|c|c|c|c|c|}
\hline Grid & 1 & 2 & 3 & 4 \\
\hline $\begin{array}{l}\text { Number of } \\
\text { Points }\end{array}$ & $65 \times 17$ & $33 \times 9$ & $17 \times 5$ & $9 \times 3$ \\
\hline
\end{tabular}

TABLE II. - VISCOUS FLOW GRID DESCRIPTIONS

\begin{tabular}{|l|c|c|c|c|c|}
\hline Grid & 1 & 2 & 3 & 4 & 5 \\
\hline $\begin{array}{l}\text { Number of } \\
\text { Points }\end{array}$ & $65 \times 33$ & $33 \times 17$ & $17 \times 9$ & $9 \times 5$ & $5 \times 3$ \\
\hline
\end{tabular}


TABLE III. - SUMMARY OF RESULTS

\begin{tabular}{|c|c|c|c|c|c|}
\hline $\begin{array}{l}\text { Test } \\
\text { Case }\end{array}$ & Coments & $\frac{\text { Thickness }}{\text { Chord }}$ & $\begin{array}{l}\text { Initial } \\
\text { Transverse } \\
\text { Spacing }\end{array}$ & $\begin{array}{l}\text { Optimal } \\
\text { Sequence } \\
\text { Length }\end{array}$ & $\begin{array}{l}\text { Work } \\
\text { Reduction } \\
\text { Factor }\end{array}$ \\
\hline $\mathbf{a}$ & $\begin{array}{l}\text { Imviscid } \\
\text { Subcritical } \\
\text { Fig. } 7\end{array}$ & 0.200 & 0.03125 & 3 & 5.1 \\
\hline $\mathbf{b}$ & $\begin{array}{l}\text { Inviscid } \\
\text { Supercritical } \\
\text { F18. } 8\end{array}$ & 0.200 & 0.03125 & 4 & 2.1 \\
\hline c & $\begin{array}{l}\operatorname{Re}=8.4 \times 10^{3} \\
\text { Laminar } \\
\text { Fig. } 9\end{array}$ & 0.000 & 0.01250 & 4 & 7.3 \\
\hline d & $\begin{array}{l}\mathrm{Re}=8.4 \times 10^{3} \\
\text { Turbulent }\end{array}$ & 0.000 & 0.01250 & 4 & 8.2 \\
\hline e & $\begin{array}{l}\operatorname{Re}=3.4 \times 10^{4} \\
\text { Laminar }\end{array}$ & 0.000 & 0.00625 & 2 & 3.1 \\
\hline f & $\begin{array}{l}\operatorname{Re}=2.0 \times 10^{5} \\
\text { Turbulent }\end{array}$ & 0.000 & 0.00250 & 2 & 2.4 \\
\hline B & $\begin{array}{l}\operatorname{Re}=8.4 \times 10^{3} \\
\text { Laminar }\end{array}$ & 0.100 & 0.01250 & 4 & 5.6 \\
\hline h & $\begin{array}{l}\text { Re }=3.4 \times 10^{4} \\
\text { Lam1nar } \\
\text { Separated } \\
\text { F18. } 10 \\
\end{array}$ & 0.100 & 0.00250 & 2 & 1.7 \\
\hline 1 & $\begin{array}{l}\operatorname{Re}=3.4 \times 10^{4} \\
\text { Turbulent } \\
\text { Separated } \\
\text { Fig. } 11\end{array}$ & 0.100 & 0.00250 & 2 & 2.1 \\
\hline 1 & $\begin{array}{l}\operatorname{Re}=3.4 \times 10^{4} \\
\text { Laminar }\end{array}$ & 0.050 & 0.00625 & 2 & 2.7 \\
\hline k & $\begin{array}{l}\text { Re }=2.0 \times 10^{5} \\
\text { Turbulent } \\
\text { F1g. } 12\end{array}$ & 0.025 & 0.00250 & 2 & 2.5 \\
\hline
\end{tabular}




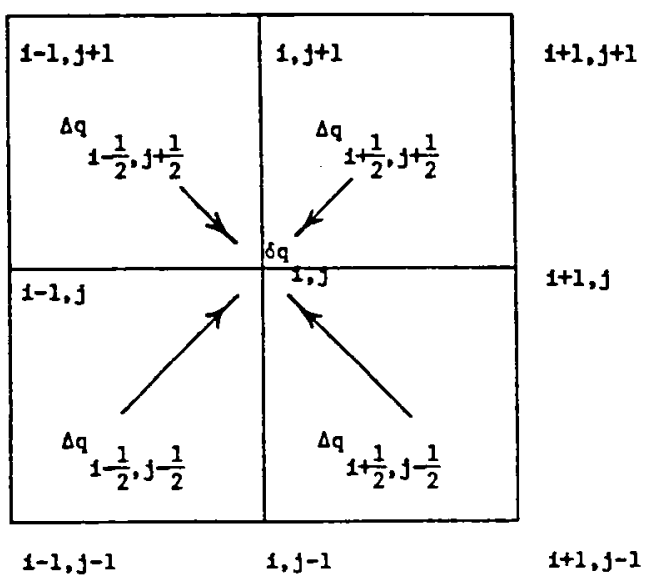

FIGURE 1. - One-S tep Lax-Wendr off Scheme

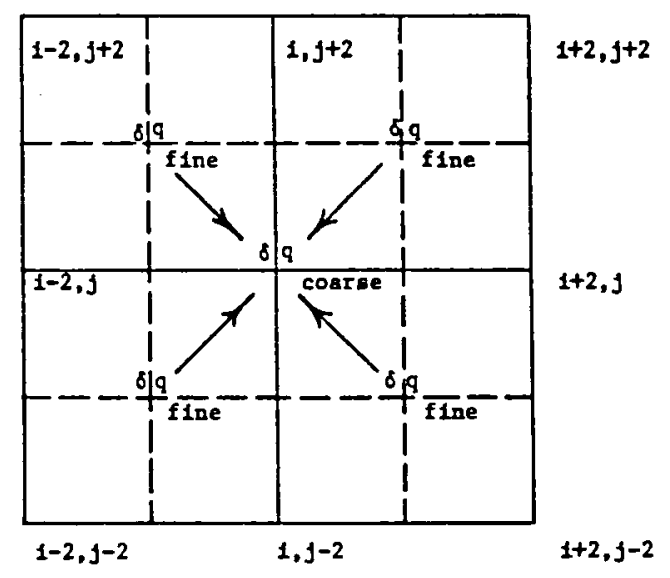

FIGURE 2. - Coarse Grid Scheme

R - Reptiction of Lateat Fine-crid oq as Coarse-Grid $\Delta q$

P - Prolongation of Coaree-crid oq to Plne Grid

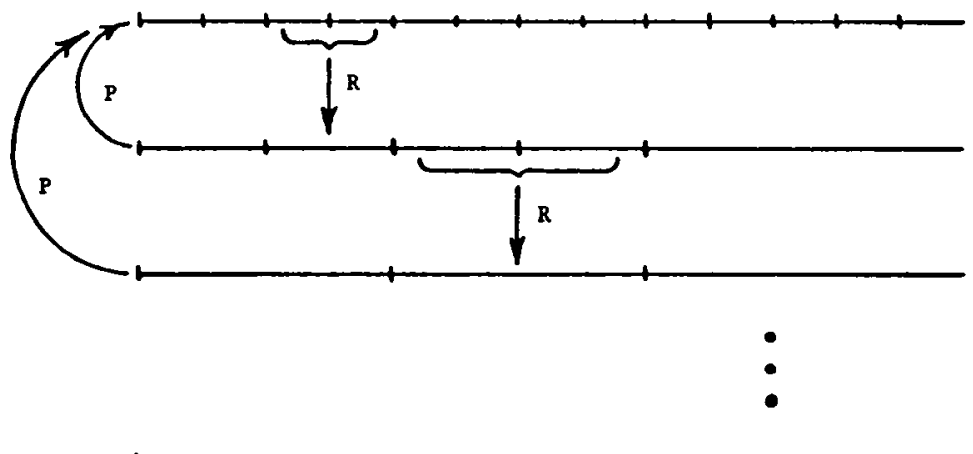

Grid

1 (Finest)

3

N (Coarseat)

FIGURE 3. - Multiple-Grid Information Flow 


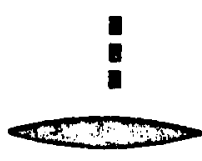

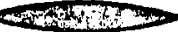

810

$\underset{\text { Direction }}{\longrightarrow}$

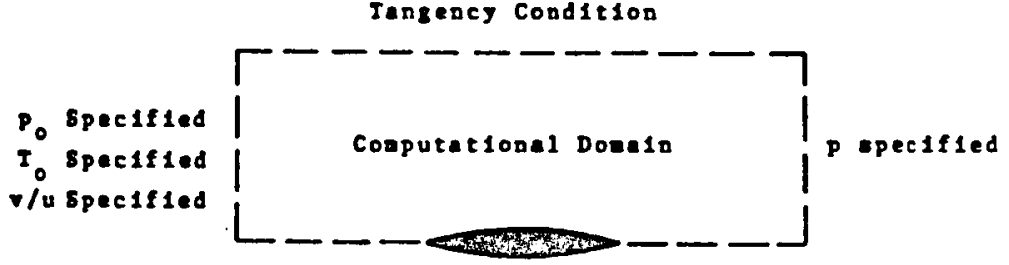

Tangency Condition

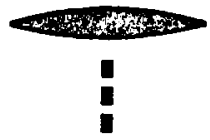

FIGURE 4. - Inviscid Bicircular Arc Cascade Problem

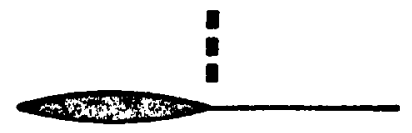

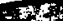
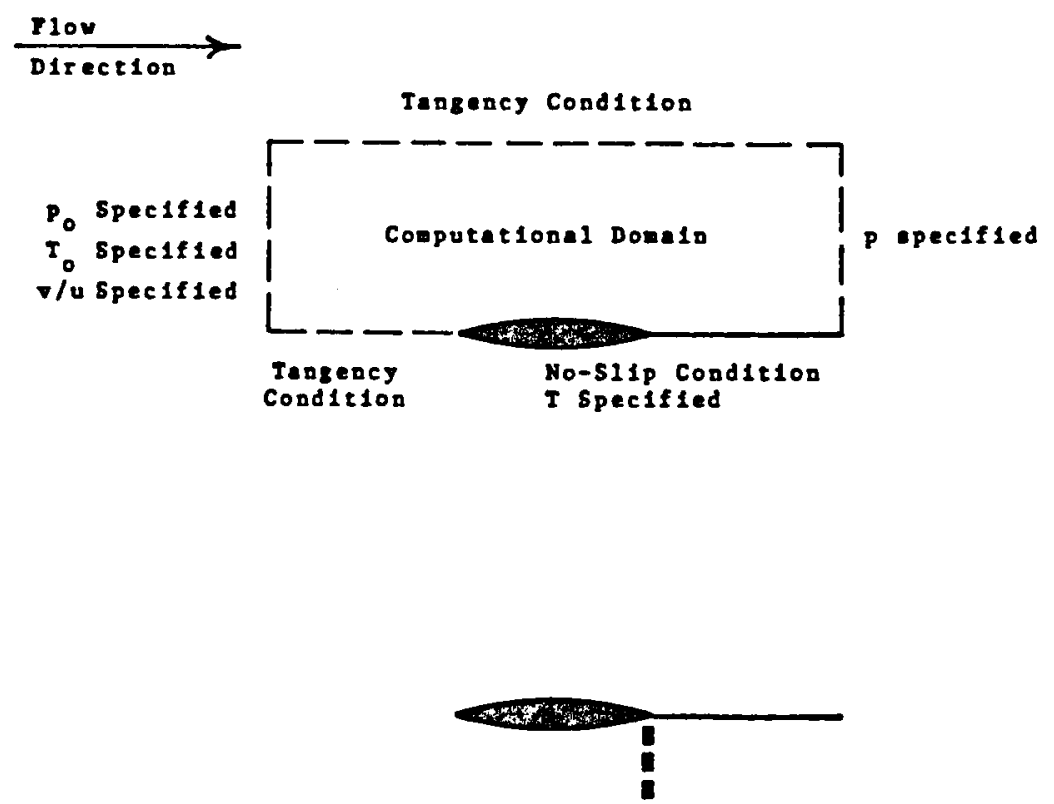

FIGURE 5. - Viscous Bicircular Arc Cascade Problem 


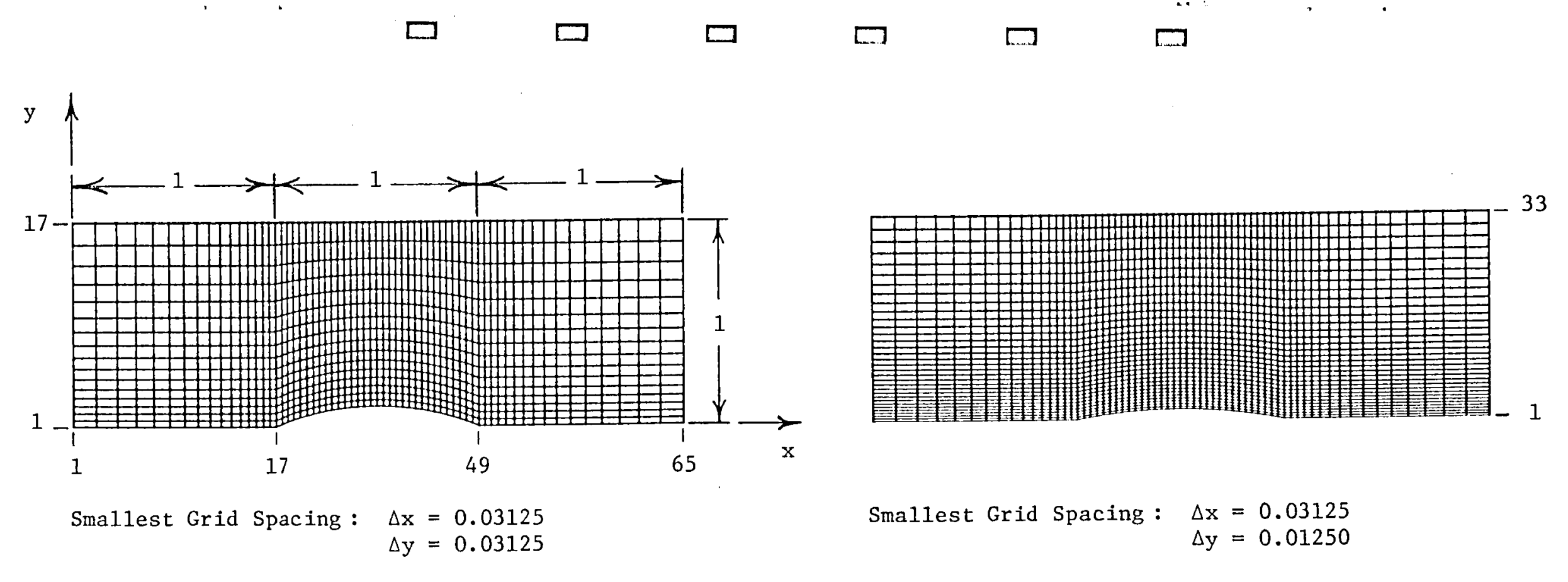

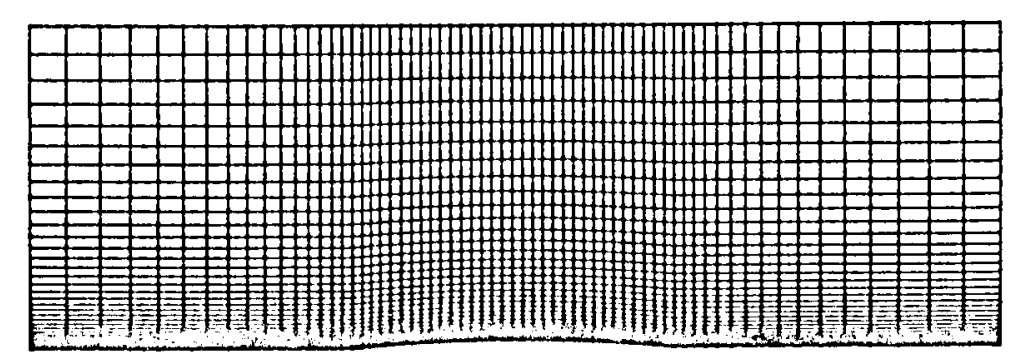

Smallest Grid Spacing : $\Delta \mathrm{x}=0.03125$

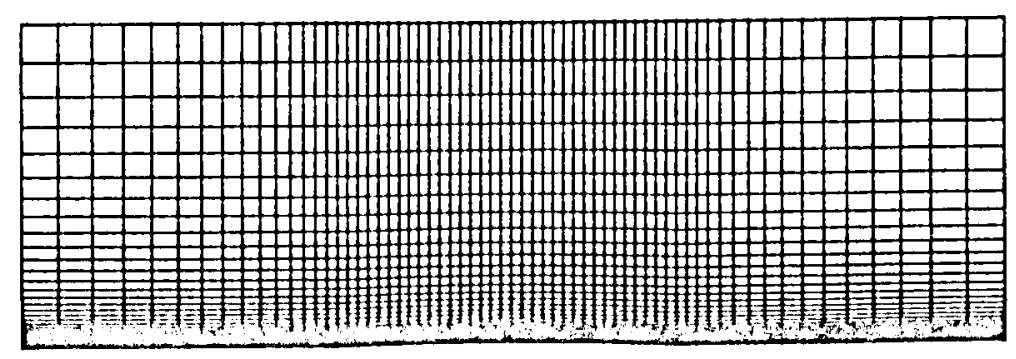

Smallest Grid Spacing : $\begin{aligned} \Delta x & =0.03125 \\ \Delta y & =0.00250\end{aligned}$

FIGURE 6. - Fine Grid Configurations 


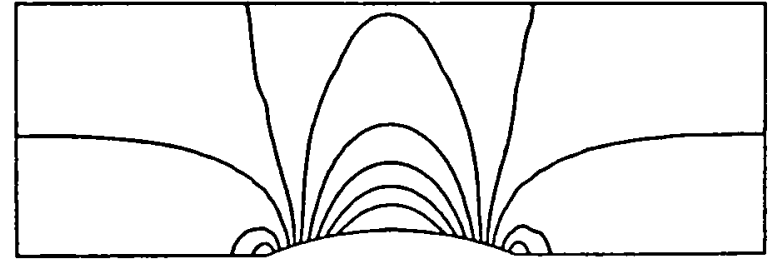

Isomachs

Convergence Histories :

Single Grid

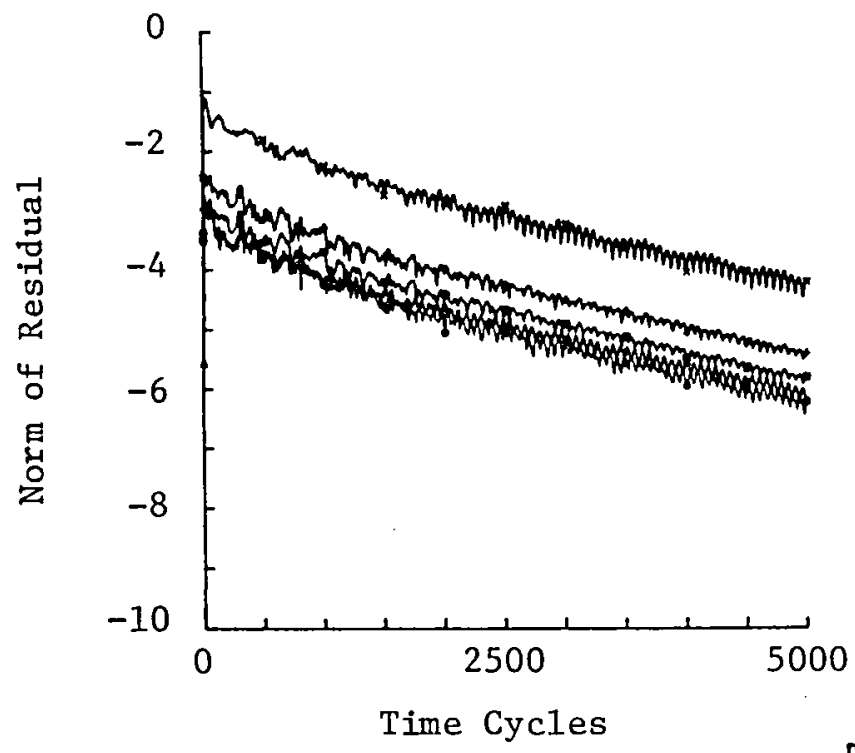

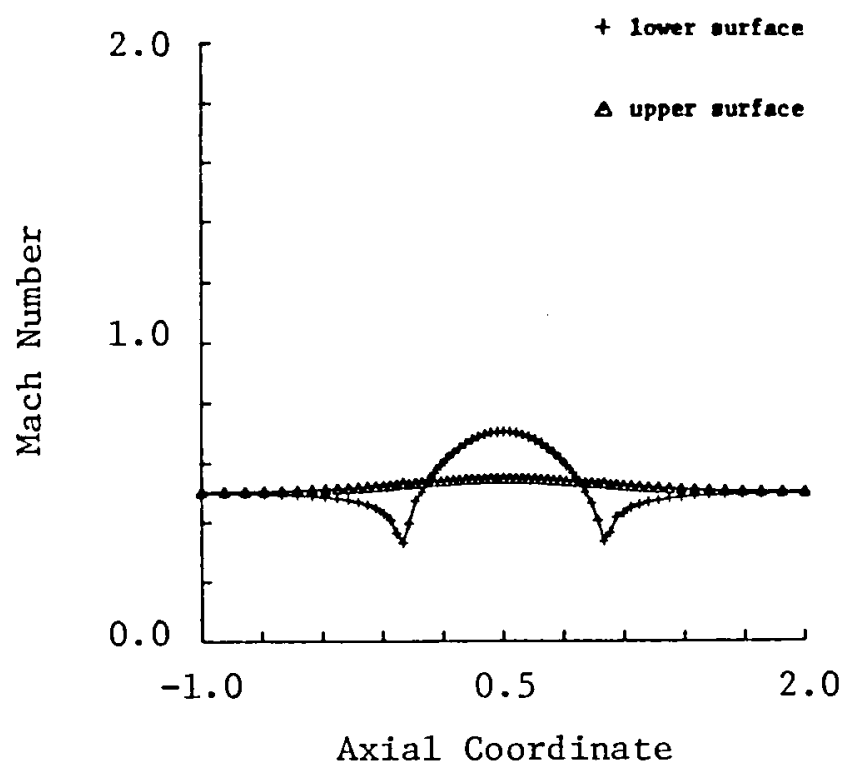

Surface Mach Number Distributions

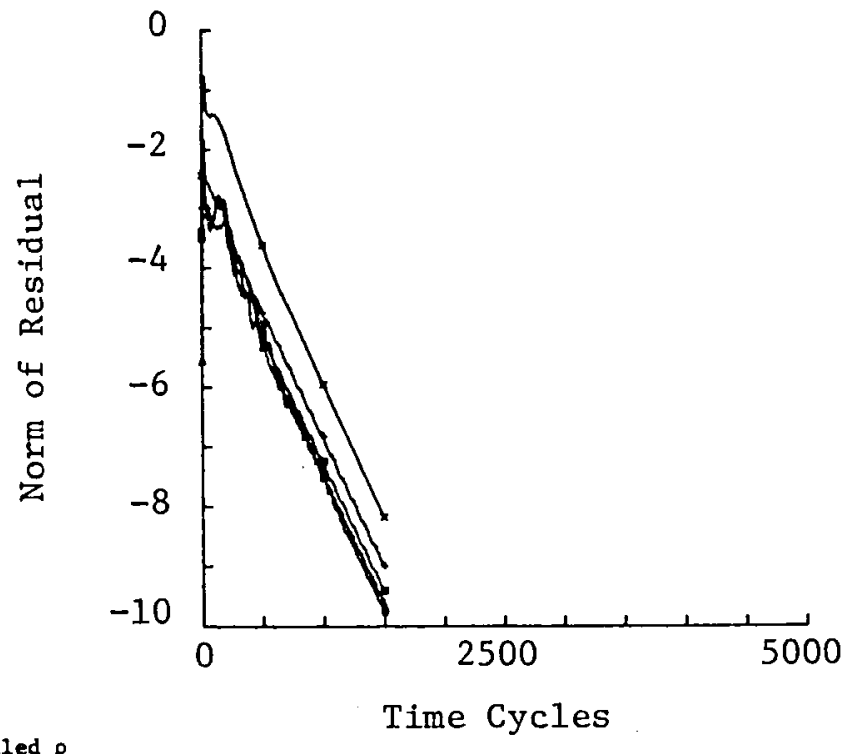

ecaled p

scaled pu

scaled pv

scaled E

X average unscaled ou

FIGURE 7. - Inviscid Subcritical Flow 


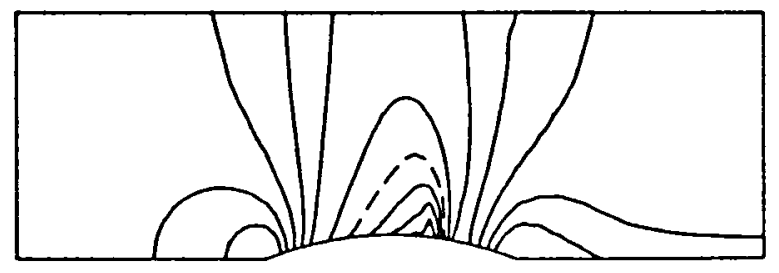

Isomachs

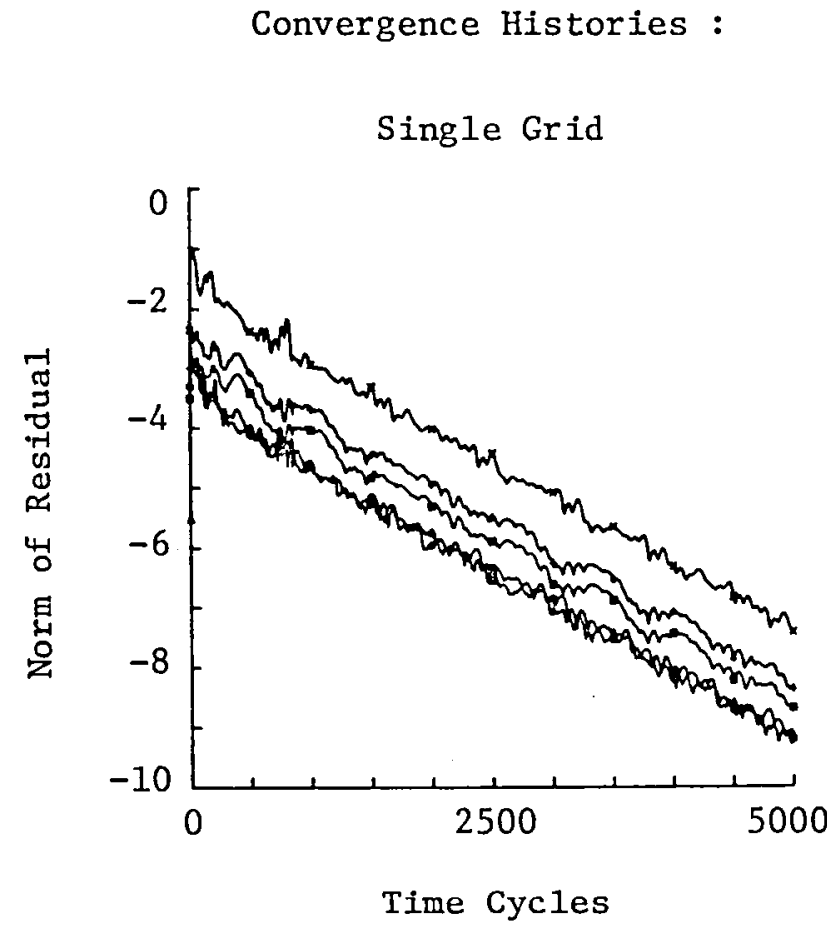

ए

- scaled ou

- ecaled ov

+ scaled E

$X$ average unsaled ou
+ lover ourface

$\Delta$ upper eurface

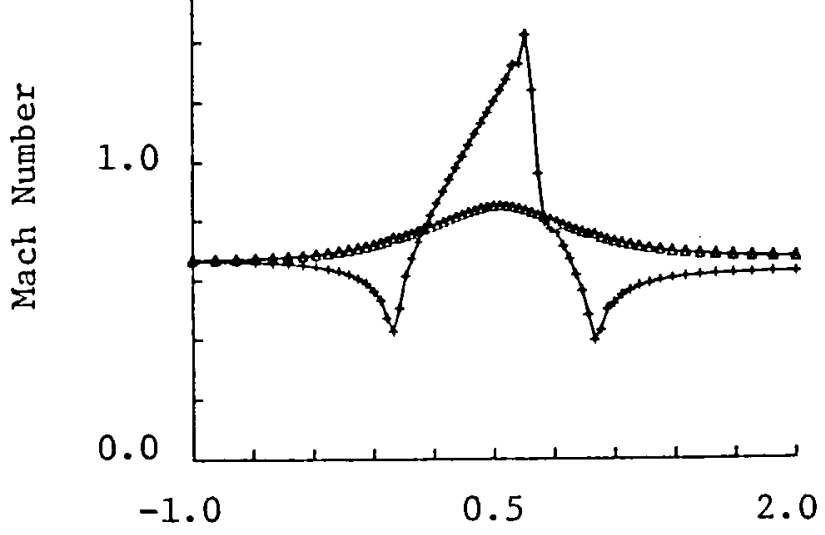

Axial Coordinate

Surface Mach Number Distributions

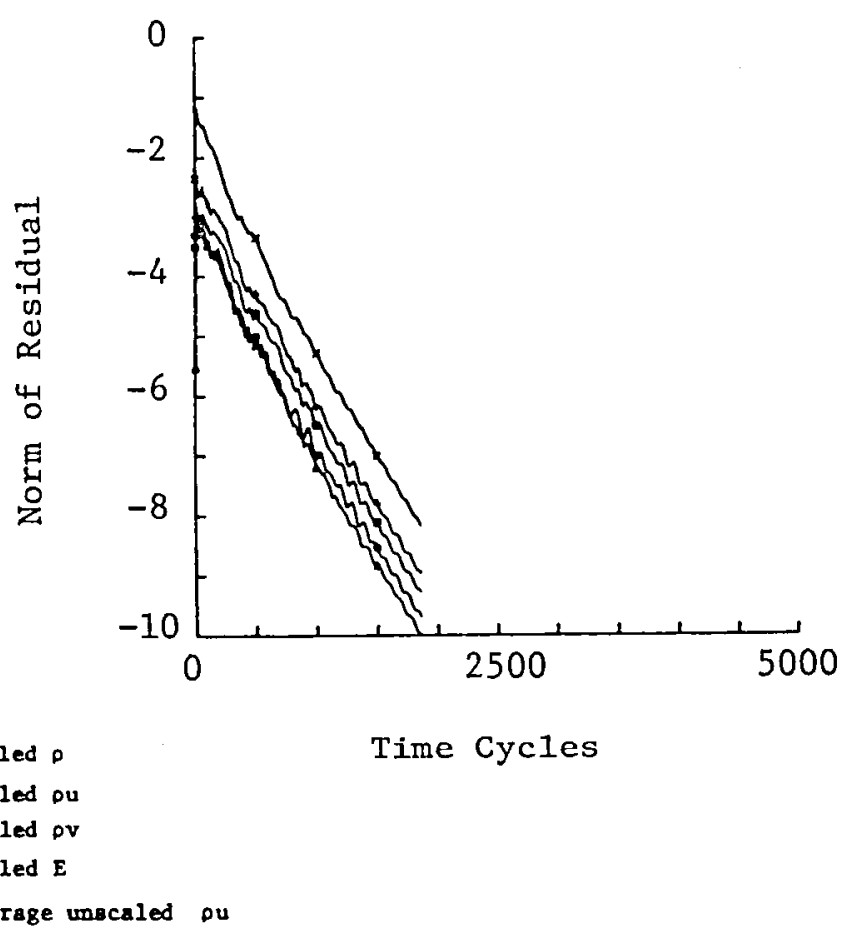

FIGURE 8. - Inviscid Supercritical Flow 


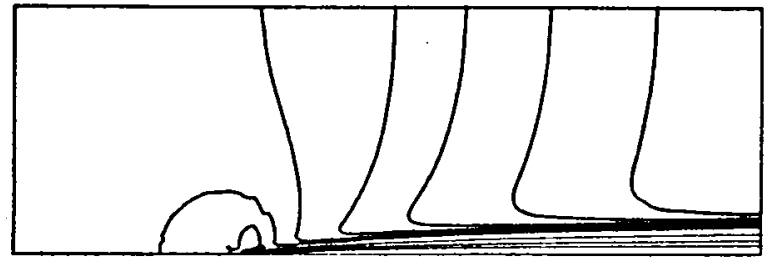

Isomachs

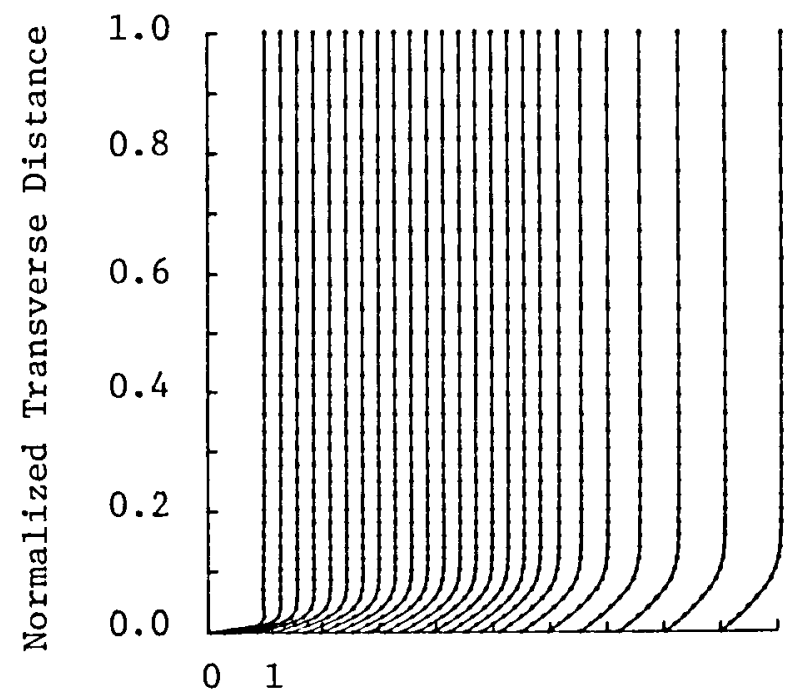

Normalized u-velocity

Velocity Profiles

Multiple Grid

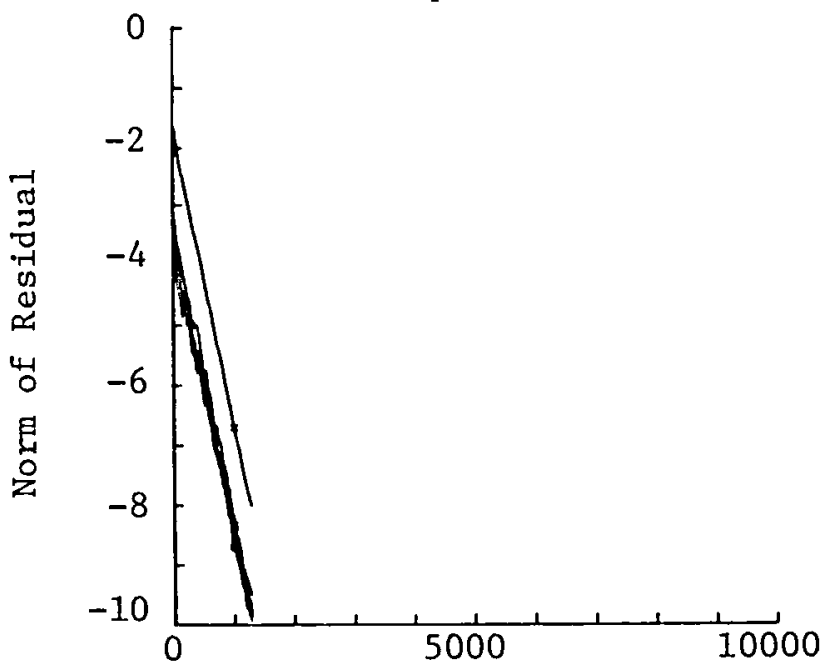

Time Cycles

\footnotetext{
$\square$ valed $\rho$

- acaled ou

- cocaled $\rho v$

+ ucaled $E$

X average unscaled ou
}

FIGURE 9. - Viscous Flow, $R e=8.4 \times 10^{3}$, Laminar 


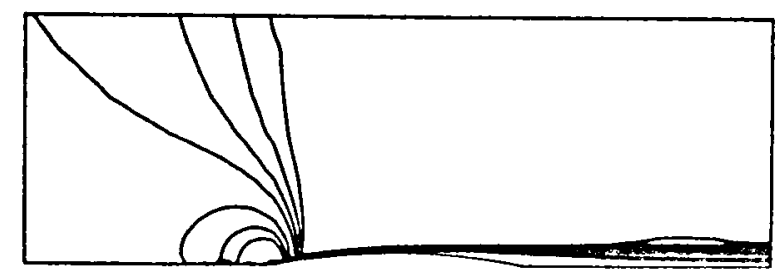

Isomachs

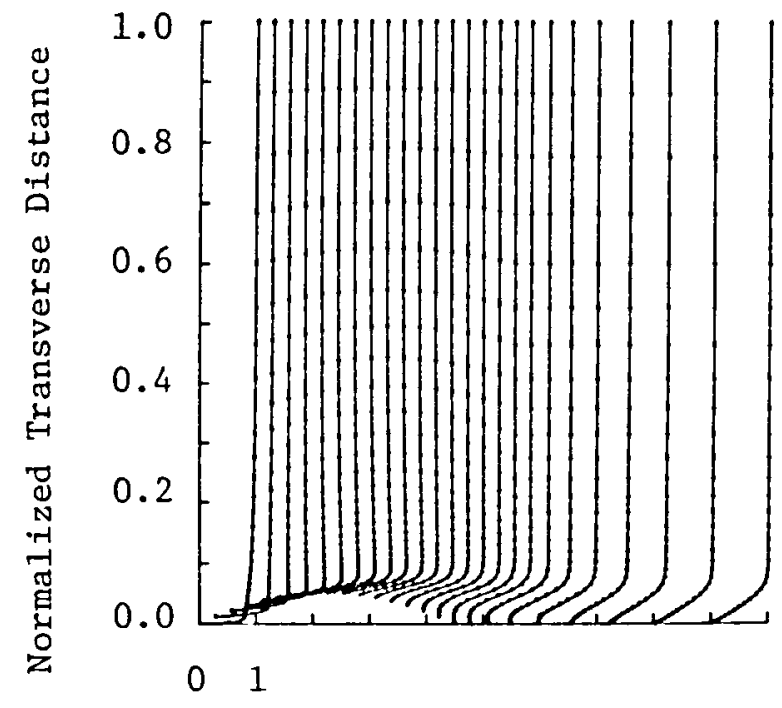

Normalized u-velocity

Velocity Profiles

Convergence Histories :

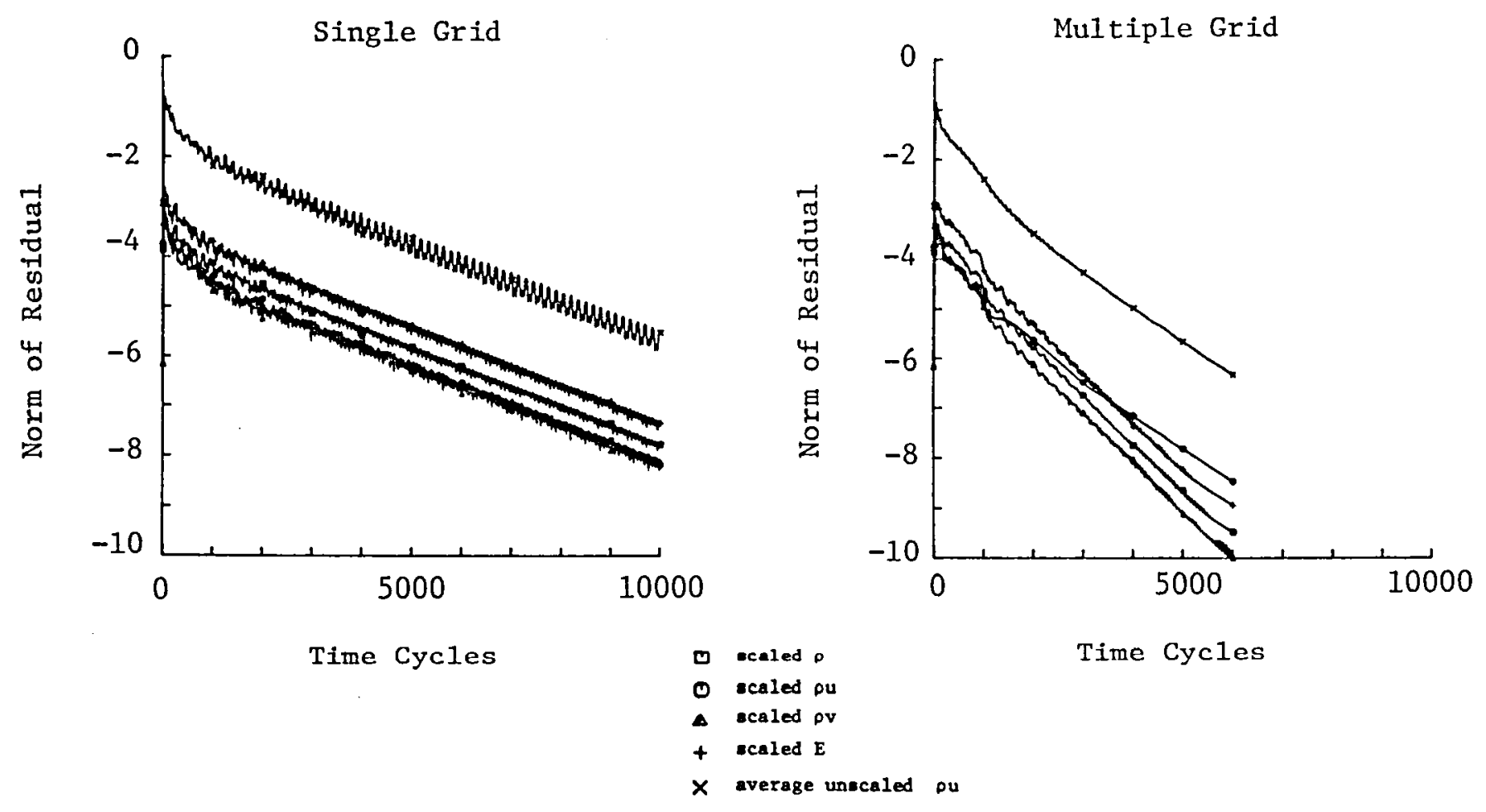

FIGURE 10. - Viscous Flow, Re $=3.4 \times 10^{4}$, Laminar, Separated 


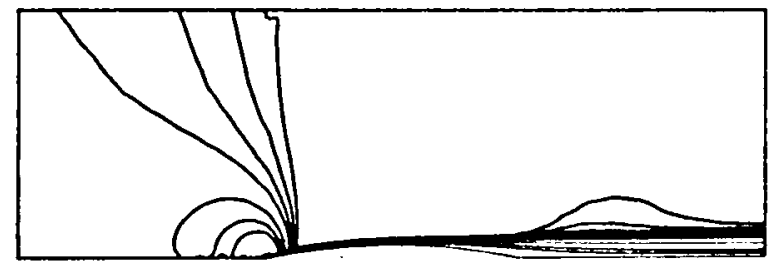

Isomachs

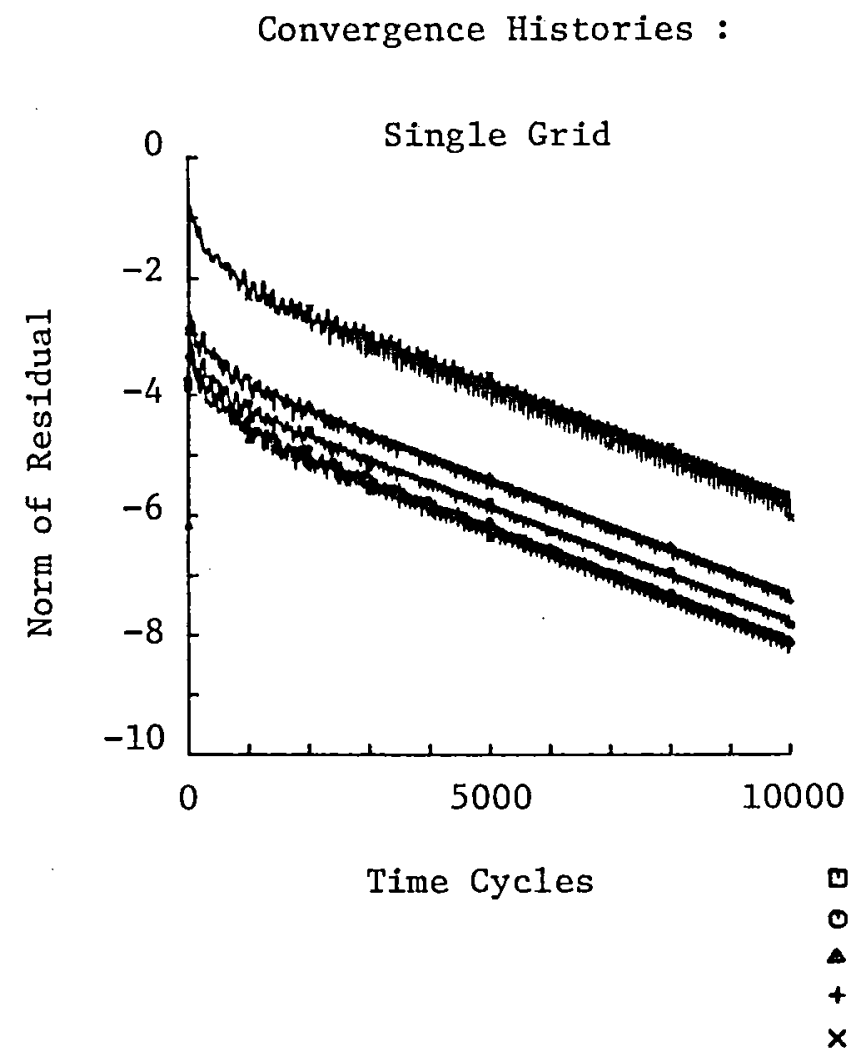

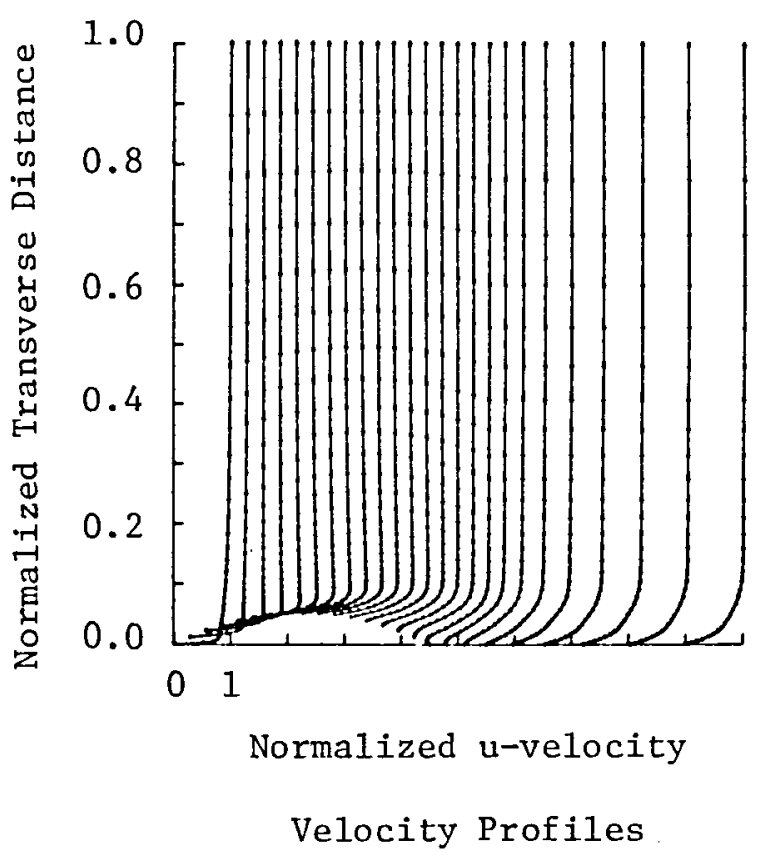

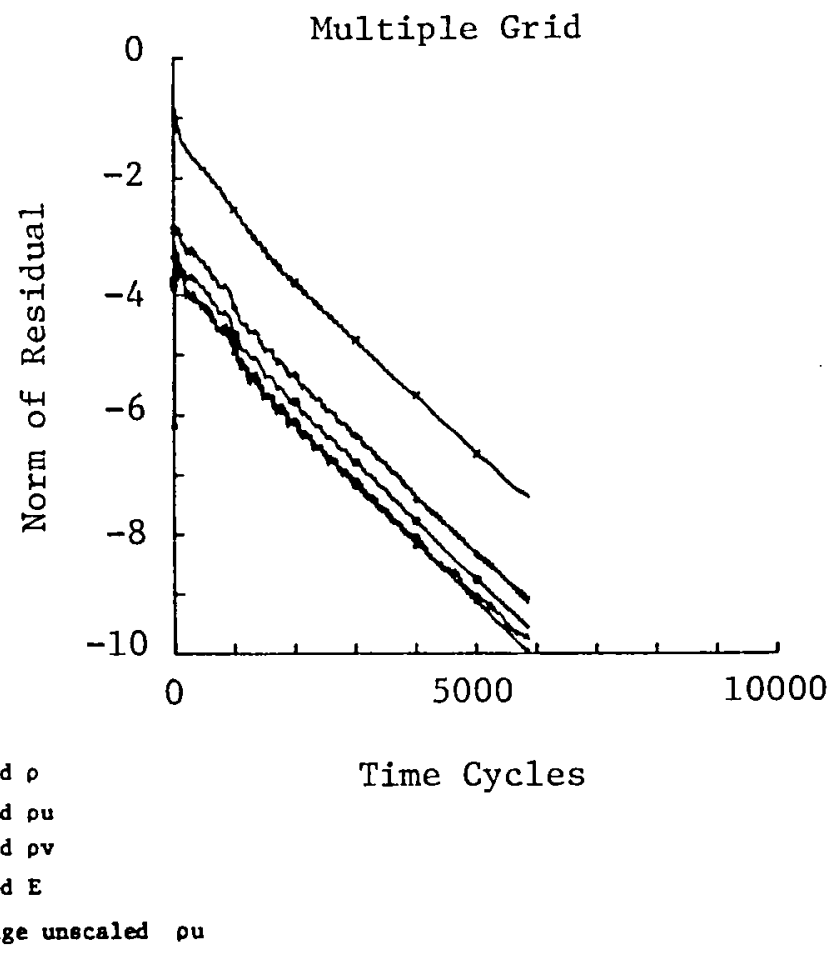

FIGURE 11. - Viscous Flow, $\mathrm{Re}=3.4 \times 10^{4}$, Turbulent, Separated 


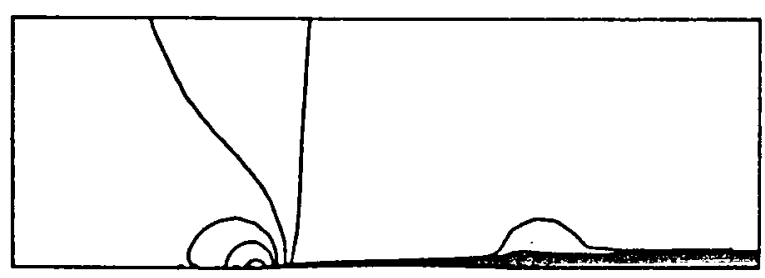

Isomachs

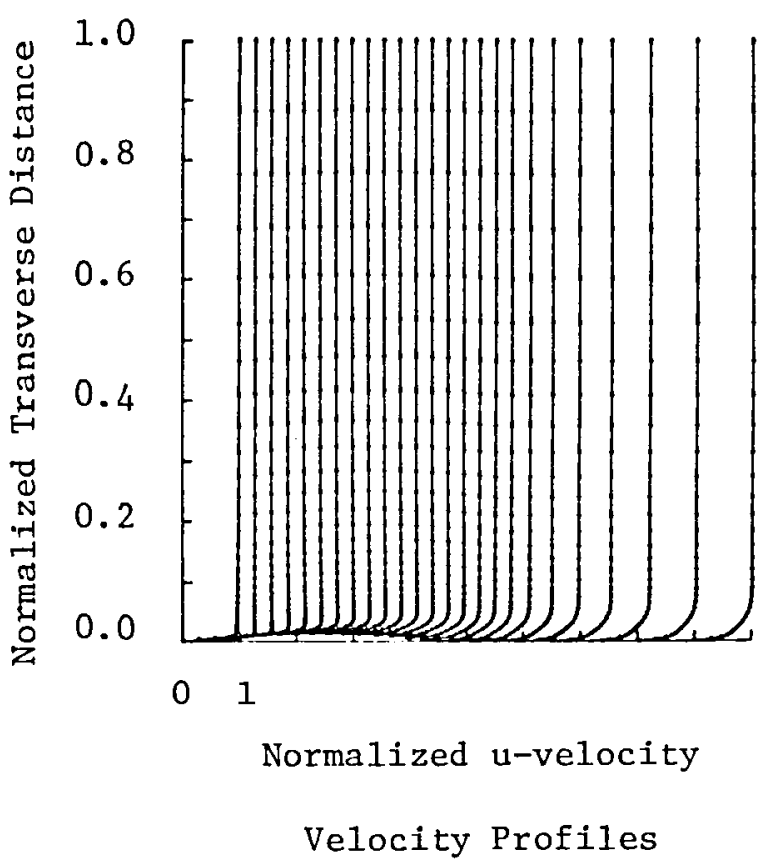

Convergence Histories :

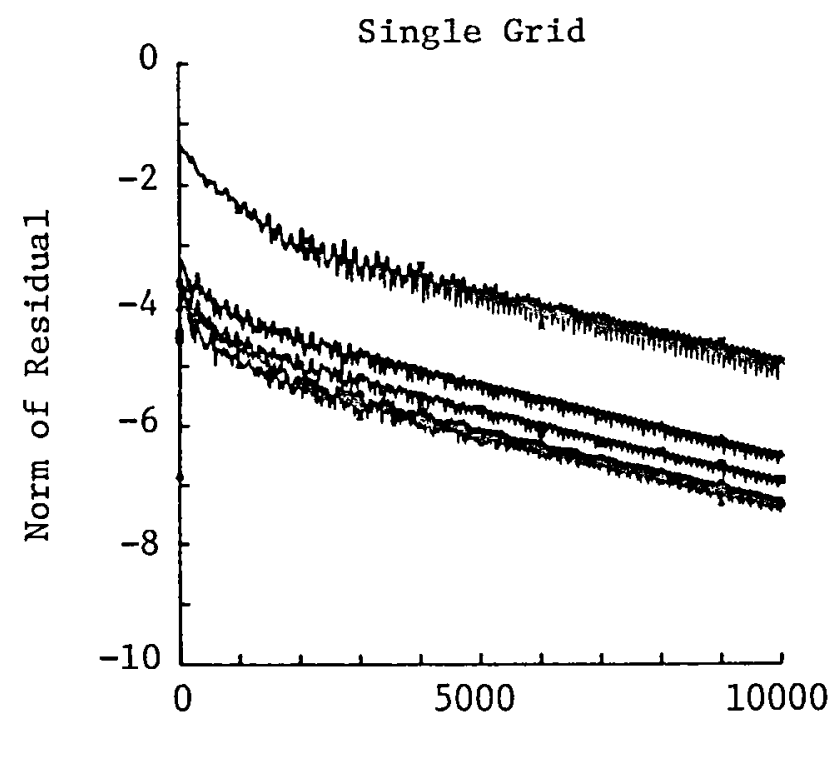

Time Cycles

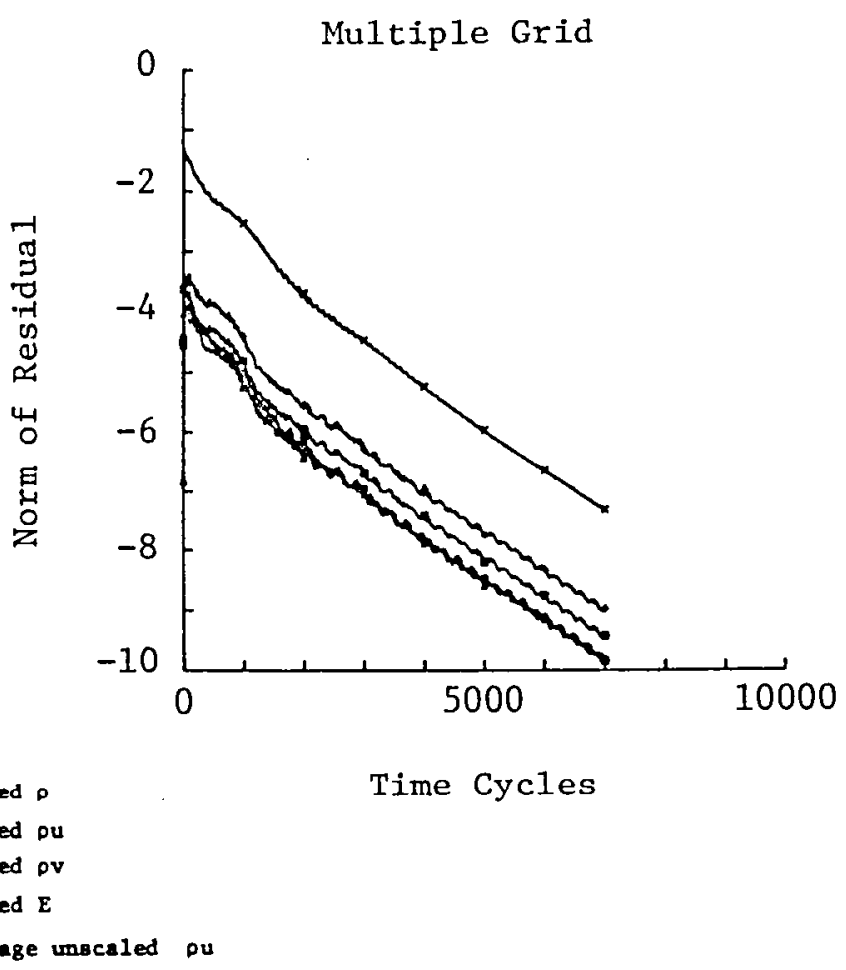

FIGURE 12. - Viscous Flow, $\operatorname{Re}=2.0 \times 10^{5}$, Turbulent 


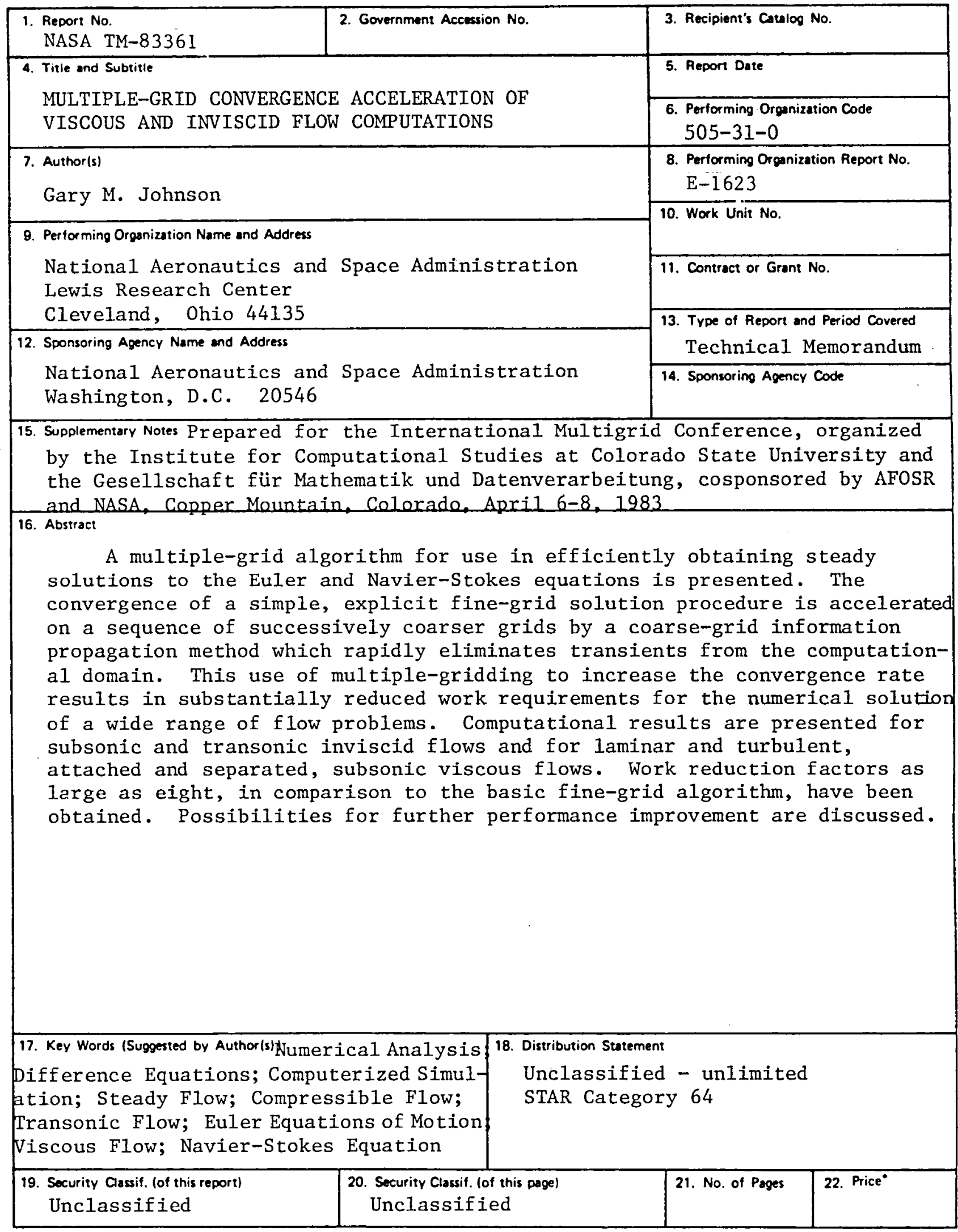

* For sale by the National Technical Information Service. Springfield. Virginia 22161 
National Aeronautics and

Space Administration

Washington, D.C.

20546

Official Business

Penalty for Private Un. $\$ 300$

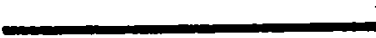

EPECIAL FOUNTH Class mail cook

उ 1176005126991

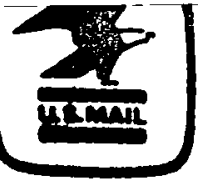

Poteces and Fees Paid Metional Aoroneutics und

Spece Administrition

Masa-45

\section{DO NOT REMOVE SLIP FROM MATERIAL}

Delete your name from this slip when returning material to the library.

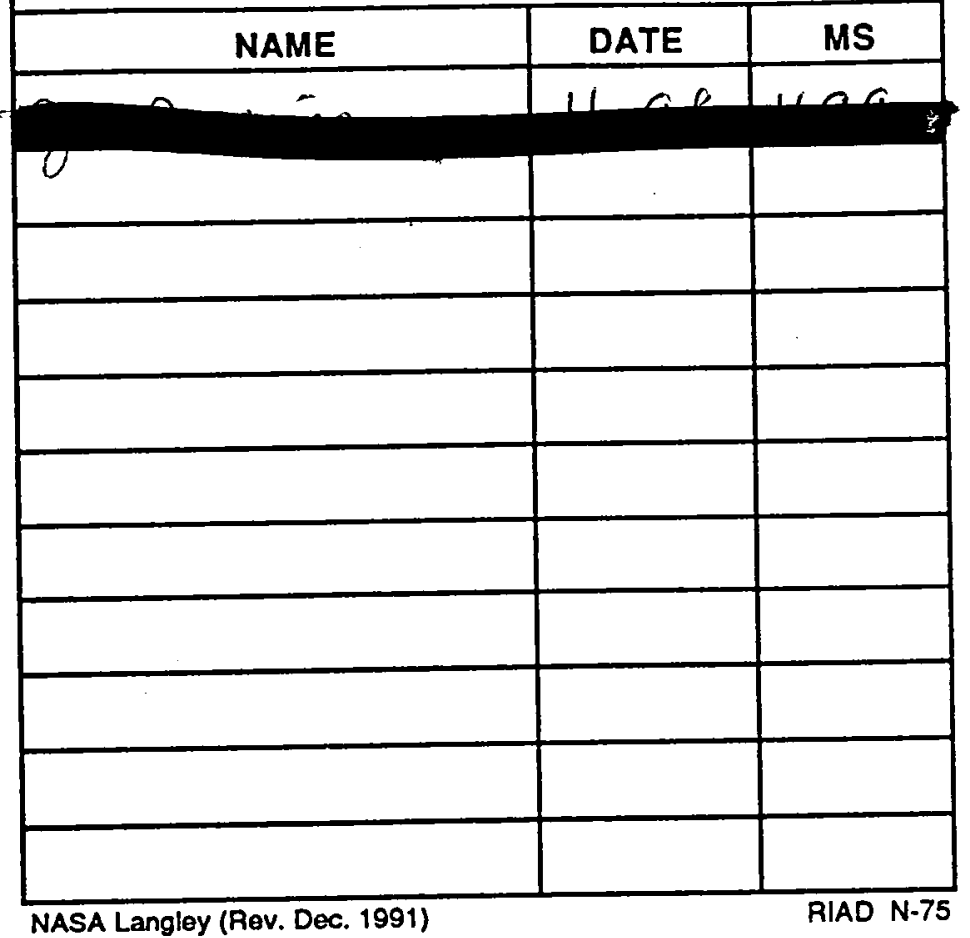

If Undelivetahle (Section $15 x$ Postal Manual) (x) Nui Return 